TRANSACTIONS OF THE

AMERICAN MATHEMATICAL SOCIETY

Volume 359, Number 9, September 2007, Pages 4325-4338

S 0002-9947(07)04165-7

Article electronically published on April 16, 2007

\title{
ON THE STRUCTURE OF NON-COMMUTATIVE WHITE NOISES
}

\author{
CLAUS KÖSTLER AND ROLAND SPEICHER
}

\begin{abstract}
We consider the concepts of continuous Bernoulli systems and non-commutative white noises. We address the question of isomorphism of continuous Bernoulli systems and show that for large classes of quantum Levy processes one can make quite precise statements about the time behaviour of their moments.
\end{abstract}

\section{INTRODUCTION}

In recent years it has become evident that looking on non-commutative algebras (in particular, operator algebras) from a stochastic point of view can be quite fruitful. So the impressive progress on our understanding of the free group von Neumann algebras relies on Voiculescu's free probability approach [Voi, VDN] and the work of Pisier and $\mathrm{Xu}$ on non-commutative martingales has opened a new direction of research PX1]. In particular, it seems that non-commutative versions of classical stochastic processes yield interesting examples of non-trivial operator-algebraic structures. At the moment we are only at the beginning of an understanding of the richness of the world of non-commutative processes; the present paper is a contribution to a systematic theory of such non-commutative processes.

Lévy processes, i.e. processes with stationary and independent processes, or 'white noises' as models for their 'derivatives', form one of the most important classes of classical stochastic processes, and the understanding of their structure was instrumental for many developments in classical probability theory. It is to be expected that the understanding of non-commutative versions of Lévy processes will be an important step towards a deeper theory of non-commutative stochastic processes.

An axiomatic frame for the treatment of non-commutative white noises was started by Kümmerer Küm1, Küm2 and is further elaborated by one of us Kös1, Kös2, HKK. Here we will address some of the canonical basic questions of this theory: namely how we can distinguish between different non-commuative white noises; and what can be said about the time behaviour of their moments. Even though a general answer to these problems for the class of all non-commutative Lévy processes seems to be out of reach (and might not even exist in this generality; see

Received by the editors November 17, 2004 and, in revised form, August 12, 2005.

2000 Mathematics Subject Classification. Primary 46L53; Secondary 46L55.

Research of the second author was supported by a Discovery Grant and a Leadership Support Initiative Award from the Natural Sciences and Engineering Research Council of Canada and by a Premier's Research Excellence Award from the Province of Ontario.

(C)2007 American Mathematical Society 
Section 4.11), we are able to provide answers to these questions for some quite large classes of non-commutative white noises.

We also want to point out that an Itô integration theory for non-commutative Lévy processes was established in [HKK]. However, our results here will not rely on this integration theory.

\section{BASIC DEFINITIONS}

We want to generalize the notion of a classical process with stationary and independent increments to a non-commutative setting. In the classical setting, it is not only the process itself which is of importance, but sometimes one is more interested in the structure of the associated filtration of $\sigma$-algebras of the increments. In the same way, we find it advantageous in the non-commutative case to distinguish between the filtration generated by the process, and the process itself. In the non-commutative setting, the filtration is given by the von Neumann algebras generated by (or, in the case of unbounded operators, affiliated to) the increments of the processes - we will address this data as a continuous Bernoulli system. We will restrict here to the finite case, i.e. where the underlying state $\varphi$ is a trace. More general definitions are possible (and desirable for a general theory), however, here we want to consider only the simplest non-trivial case.

Notation 2.1. By $\mathcal{I}$ we denote the set of all intervals $I \subset \mathbb{R}$ of the form $I=[s, t)$ for $-\infty<s<t<\infty$. For $I=[s, t) \in \mathcal{I}$ and $u \in \mathbb{R}$ we denote by $I+u$ the interval

$$
I+u:=[s+u, t+u) .
$$

Definition 2.2. A (non-commutative) continuous Bernoulli system $\left(\mathcal{A}, \varphi,\left(\mathcal{A}_{I}\right)_{I \in \mathcal{I}}\right)$ consists of

(i) a non-commutative probability space $(\mathcal{A}, \varphi)$, where $\mathcal{A}$ is a von Neumann algebra with separable predual and $\varphi$ is a faithful and normal trace $\varphi$ on $\mathcal{A}$

(ii) a filtration $\left(\mathcal{A}_{I}\right)_{I \in \mathcal{I}}$, where $\mathcal{A}_{I}$ are von Neumann subalgebras of $\mathcal{A}$ such that the following properties are satisfied:

(a) global minimality:

$$
\mathcal{A}=\bigvee\left\{\mathcal{A}_{I} \mid I \in \mathcal{I}\right\}
$$

(b) isotony:

$$
\mathcal{A}_{I} \subset \mathcal{A}_{J} \quad \text { whenever } I \subset J
$$

(c) $\mathbb{C}$-independence: for all $I, J \in \mathcal{I}$ with $I \cap J=\emptyset$ we have that

$$
\varphi(a b)=\varphi(a) \varphi(b)
$$

for all $a \in \mathcal{A}_{I}$ and all $b \in \mathcal{A}_{J}$.

Remarks 2.3. 1) Notice that we can also phrase the $\mathbb{C}$-independence in the following form: for all $s<t<u$,

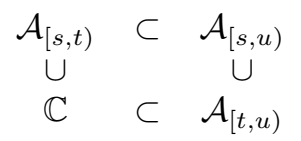

is a (not necessarily non-degenerate) commuting square $\mathrm{Pop}$. If the von Neumann algebra $\mathcal{A}$ is commutative, $\mathbb{C}$-independence is equivalent to the usual notion of stochastic independence in probability theory. 
2) In our tracial frame, $\mathbb{C}$-independence is clearly equivalent to pyramidal independence: for all $I, J \in \mathcal{I}$ with $I \cap J=\emptyset$ we have that

$$
\varphi\left(a_{1} b a_{2}\right)=\varphi\left(a_{1} a_{2}\right) \varphi(b) \quad \text { for all } a_{1}, a_{2} \in \mathcal{A}_{I} \text { and all } b \in \mathcal{A}_{J} .
$$

In a more general, non-tracial frame, one needs the existence of conditional expectations to ensure pyramidal independence.

3) Time-homogenity of our processes on the level of continuous Bernoulli systems can be encoded via the requirement of the existence of a shift which is compatible with the filtration - in this case we call such a system a non-commutative continuous Bernoulli shift. These shifts are introduced in HKK and provide a non-commutative extension of Tsirelson's noises or homogeneous continuous product systems of probability spaces Tsi. Similarly, continuous Bernoulli systems are a non-commutative extension of continuous products of probability spaces.

The definition of the notion 'continuous Bernoulli system' puts the whole emphasis on the von Neumann algebras without specifying an underlying process with independent increments. In our setting the information about the increments of the process will be encoded in the notion of an additive flow. As the example of classical Brownian motion shows, the increments do not need to consist of bounded operators, thus these flows need not be elements of the von Neumann algebras. In general, they will be given by closed densely defined affiliated operators. In the following we will restrict ourselves to the case where these flows are elements of non-commutative $L^{p}$-spaces, such that all moments of the flow exist.

Notation 2.4. Let $\mathcal{A}$ be a von Neumann algebra and $\varphi$ a faithful normal trace. For $1 \leq p<\infty$, the non-commutative $L^{p}$-spaces $L^{p}(\mathcal{A})$ are defined as the completion of $\mathcal{A}$ in the norm

$$
\|x\|_{p}:=\left(\varphi\left(|x|^{p}\right)\right)^{1 / p} \quad(x \in \mathcal{A}),
$$

where $|x|=\left(x^{*} x\right)^{1 / 2} . L^{\infty}(\mathcal{A})$ is just $\mathcal{A}$ with the usual operator norm. Furthermore, we put

$$
L^{\infty-}(\mathcal{A}):=\bigcap_{1 \leq p<\infty} L^{p}(\mathcal{A}) .
$$

Notice that $\varphi$ extends from $\mathcal{A}$ to $L^{1}(\mathcal{A})$ and that this extension will be denoted by the same symbol $\varphi$. For further details on non-commutative $L^{p}$-spaces we refer to PX2 and the literature cited therein.

Definition 2.5. Let $\left(\mathcal{A}, \varphi,\left(\mathcal{A}_{I}\right)_{I \in \mathcal{I}}\right)$ be a continuous Bernoulli system. An additive flow (more precisely, additive $L^{\infty-}$-flow) is a family $B=\left(B_{I}\right)_{I \in \mathcal{I}} \subset L^{\infty-}(\mathcal{A})$ such that we have

(i) continuity: the map

$$
(s, t) \mapsto B_{[s, t)} \in L^{p}(\mathcal{A})
$$

is, for all $1 \leq p<\infty$, jointly continuous in $s$ and $t$;

(ii) adaptedness: $B_{I} \in L^{\infty-}\left(\mathcal{A}_{I}\right)$ for all $I \in \mathcal{I}$;

(iii) additivity: $B_{[s, u)}=B_{[s, t)}+B_{[t, u)}$ for all $s<t<u$.

If $\varphi\left(B_{I}\right)=0$ for all $I \in \mathcal{I}$, then we call the flow centred. If $B_{I} \subset \mathbb{C} 1$ for all $I \in \mathcal{I}$, then the flow is trivial. A normalized flow is centred and satisfies $\varphi\left(B_{[0,1)}^{*} B_{[0,1)}\right)=1$. 
A flow $\left(B_{I}\right)_{I \in \mathcal{I}}$ is stationary if we have the invariance of all its moments in the following sense: for all $n \in \mathbb{N}$ and all $I_{1}, \ldots, I_{n} \in \mathcal{I}$ we have that $\varphi\left(B_{I_{1}+t} \ldots B_{I_{n}+t}\right)$ does not depend on $t \in \mathbb{R}$.

For a given stationary flow $\left(B_{I}\right)_{I \in \mathcal{I}}$ we put

$$
B_{t}:=B_{[0, t)} \quad(t>0), \quad B_{0}:=0
$$

and call $\left(B_{t}\right)_{t \geq 0}$ the corresponding quantum Lévy process.

Remarks 2.6. 1) Notice that we can always turn an additive flow $B_{I}$ into a centred additive flow by considering $B_{I}-\varphi\left(B_{I}\right)$.

2) From stationarity and continuity it follows that we have for a stationary centered flow that (see also Lemma 4.1)

$$
\varphi\left(B_{t}^{2}\right)=\varphi\left(B_{1}^{2}\right) \cdot t
$$

In the same way, by also invoking the independence of increments, we get that for any two stationary centered flows $\left(B_{I}\right)_{I \in \mathcal{I}}$ and $\left(\tilde{B}_{I}\right)_{I \in \mathcal{I}}$ we have

$$
\varphi\left(B_{[0, t)} \tilde{B}_{[0, t)}\right)=\varphi\left(B_{[0,1)} \tilde{B}_{[0,1)}\right) \cdot t .
$$

In particular, for a normalized stationary flow we have

$$
\varphi\left(B_{[0, t)} B_{[0, t)}^{*}\right)=t .
$$

3) Note that we can recover our stationary flow from the quantum Lévy process via

$$
B_{[s, t)}=B_{t}-B_{s} .
$$

This gives $B_{I}$ only for $I \subset \mathbb{R}_{+}$, however, in the stationary case this contains all essential information. Thus, stationary flows and quantum Lévy processes are just two sides of the same object.

In most concrete cases, continuous Bernoulli systems are given as von Neumann algebras generated by specified quantum Lévy processes. However, there exist examples of continuous Bernoulli systems without any non-trivial quantum Lévy process (see also [HKK, Theorems 4.4.3 and 6.5.8]). In analogy with the classification of product systems of Hilbert spaces such examples might be addressed as non-type I. We are here mainly interested in type I, i.e. those having stationary flows which generate the von Neumann algebras. From a probabilistic point of view it seems to be appropriate to call such type I continuous Bernoulli systems non-commutative white noises (see also [HKK, Subsection 6.5] for the time-homogeneous setting). The question of continuous Bernoulli systems without non-trivial flows and the relation of the present frame with the work of Arveson [Arv and Tsirelson Tsi] on product systems will be discussed elsewhere Kös3.

\section{IsOMORPHISM OF CONTINUOUS BERNOULLI SYSTEMS}

A first canonical problem is to classify continuous Bernoulli systems modulo a notion of isomorphism which respects the filtration.

Definition 3.1. We say that two continuous Bernoulli systems $\left(\mathcal{A}, \varphi,(\mathcal{A})_{I \in \mathcal{I}}\right)$ and $\left(\mathcal{B}, \psi,(\mathcal{B})_{I \in \mathcal{I}}\right)$ are isomorphic, if there exists an isomorphism $\pi: \mathcal{A} \rightarrow \mathcal{B}$ which 
respects the filtration, i.e.

$$
\pi\left(\mathcal{A}_{I}\right)=\mathcal{B}_{I} \quad \text { for all } I \in \mathcal{I},
$$

and such that

$$
\varphi=\psi \circ \pi
$$

We will call such a $\pi$ filtration preserving.

Note that the latter condition on the traces is automatically fulfilled if the von Neumann algebras $\mathcal{A}$ and $\mathcal{B}$ are factors.

This isomorphism problem asks for a classification of subfactors of von Neumann algebras in the extreme case where we have a continuous family of subalgebras (of necessarily infinite index).

Since a filtration preserving isomorphism extends to isometries between the corresponding $L^{p}$-spaces $(1 \leq p<\infty)$, normalized stationary flows are mapped to normalized stationary flows. Thus it is clear that the set of all distributions of such flows yields an invariant for filtration preserving isomorphisms. In particular, if we have only one such distribution, then this can be used to distinguish different continuous Bernoulli systems.

The uniqueness of such a distribution is, for example, given in the case of classical Brownian motion. One way to see this is to invoke the chaos decomposition property of the $L^{2}$-space of classical Brownian motion. This says that every element in the $L^{2}$-space can be represented (in a unique way) as a sum of multiple Wiener integrals with respect to Brownian motion. This means in particular that every flow can be represented in terms of multiple integrals, and by using the stationarity and the independence of the increments this readily implies that such a stationary flow has to have Gaussian distributions.

We want to imitate that argument in the non-commutative case. The chaos decomposition of the $L^{2}$-space into multiple Wiener integrals equips the $L^{2}$-space with a Fock space structure $\bigoplus L^{2}\left(\mathbb{R}^{n}\right)$, and the main argument then consists of the simple observation that non-trivial flows exist in $L^{2}\left(\mathbb{R}^{n}\right)$ only for $n=1$.

As it turns out, in general we do not have such a chaos decomposition of the $L^{2}$-space of a given continuous Bernoulli system. Even if we restrict to noncommutative versions of Brownian motions this chaos decomposition is not present in general. However, for a quite big class of continuous Bernoulli systems we have a more general kind of chaos decomposition for the corresponding $L^{2}$-space, resembling a Fock space decomposition, but carrying some additional information.

The class of continuous Bernoulli systems for which such a more general kind of chaos decomposition is available are the so-called generalized Brownian motions, which were introduced in BSp2. They are characterized by the requirement that all mixed moments in such a Brownian motion can be calculated by a kind of Wick formula in terms of a given function $\mathbf{t}$ on pair partitions. In GM2, Guta and Maassen have shown that this class of generalized Brownian motions coincides with the class of operators arising in their construction GM1 of symmetric Hilbert spaces in terms of the combinatorial concept of species. In particular, they provide a concrete realization of the $L^{2}$-space of the generalized Brownian motions. Namely, they are of a Fock space like form $\mathcal{F}_{V}(\mathcal{H})$, carrying, however, in general some additional information, which is encoded in a sequence $V=\left(V_{n}\right)_{n=0}^{\infty}$ of (not necessarily finite dimensional) Hilbert spaces such that each $V_{n}$ carries a unitary 
representation $U_{n}$ of the symmetric group $S(n)$. Then

$$
\mathcal{F}_{V}(\mathcal{H}):=\bigoplus_{n=0}^{\infty} \frac{1}{n !} V_{n} \otimes_{s} \mathcal{H}^{\otimes n}
$$

i.e. $\mathcal{F}_{V}(\mathcal{H})$ is spanned by linear combinations of vectors of the form

$$
v \otimes_{s} h_{1} \otimes \cdots \otimes h_{n}:=\frac{1}{n !} \sum_{\pi \in S(n)} U_{n}(\pi) v \otimes \tilde{U}_{n}(\pi) h_{1} \otimes \cdots \otimes h_{n},
$$

where $\tilde{U}_{n}$ is the canonical action of $S(n)$ on the $n$-fold tensor product of $\mathcal{H}$.

The concrete structure of the space $\mathcal{F}_{V}$ depends of course on the scalar product in the spaces $V_{n}$, which is determined by the underlying function $\mathbf{t}$. Of course, this Fock space structure is compatible with the filtration $I \mapsto L^{2}(I)$, i.e. under the identification of $L^{2}(\mathcal{A})$ with $\mathcal{F}_{V}\left(L^{2}(\mathbb{R})\right)$, the subspace $L^{2}\left(\mathcal{A}_{I}\right)$ is, for each $I \in \mathcal{I}$, mapped onto $\mathcal{F}_{V}\left(L^{2}(I)\right)$.

It is this form of decomposition for the $L^{2}$-space which gives restrictions for a flow. Although some of the following arguments might be extended to more general situations, we will, for sake of clarity, restrict ourselves to the (quite big!) class of generalized Brownian motions. In the following we will denote by $L_{\text {loc }}^{2}$ the set of locally $L^{2}$-functions, i.e. those measurable functions whose restriction to any compact interval is $L^{2}$.

Theorem 3.2. Let $\left(\mathcal{A}, \varphi,\left(\mathcal{A}_{I}\right)_{I \in \mathcal{I}}\right)$ be a continuous Bernoulli system, generated by a generalized Brownian motion, with generalized chaos decomposition $L^{2}(\mathcal{A})=$ $\mathcal{F}_{V}\left(L^{2}(\mathbb{R})\right)$ for $V=\left(V_{0}, V_{1}, \ldots\right)$. Then the set of centered flows for $\left(\mathcal{A}, \varphi,\left(\mathcal{A}_{I}\right)_{I \in \mathcal{I}}\right)$ can be identified with the one-particle space $V_{1} \otimes_{s} L_{\text {loc }}^{2}(\mathbb{R}) \cong L_{\text {loc }}^{2}\left(\mathbb{R}, V_{1}\right)$, via

$$
L_{l o c}^{2}\left(\mathbb{R}, V_{1}\right) \ni \xi \mapsto\left(B_{I}(\xi)\right)_{I \in \mathcal{I}},
$$

where

$$
B_{I}(\xi):=\xi \cdot \chi_{I} .
$$

Proof. It is clear that any $\left(B_{I}(\xi)\right)_{I \in \mathcal{I}}$ is a centered flow. (Note that all moments of these operators exist by the construction of generalized Brownian motions and that they are continuous in the endpoints of the intervals $I$.)

For the other direction, consider a centered flow $\left(B_{I}\right)_{I \in \mathcal{I}}$. Since, by definition, all its moments exist, we must have that $B_{I} \in \mathcal{F}_{V}\left(L^{2}(I)\right)$. We decompose $B_{I}$ according to the direct sum decomposition of our $L^{2}$-space as

$$
B_{I}=\bigoplus_{n=0}^{\infty} B_{I}^{(n)} \quad \text { with } \quad B_{I}^{(n)} \in \frac{1}{n !} V_{n} \otimes_{s} L^{2}(I)^{\otimes n} \subset V_{n} \otimes L^{2}\left(I^{n}\right) .
$$

Note that each $\left(B_{I}^{(n)}\right)_{I \in \mathcal{I}}$ is a flow, too. Fix $I \in \mathcal{I}$ and decompose it, for each $N \in \mathbb{N}$, into the disjoint union of intervals $I_{N, 1}, \ldots, I_{N, N}$ of the same length. Then, for each $n \in \mathbb{N}$, we have

$$
B_{I}^{(n)}=B_{I_{N, 1}}^{(n)}+\cdots+B_{I_{N, N}}^{(n)} \subset V_{n} \otimes\left(L^{2}\left(I_{N, 1}^{n}\right) \cup \cdots \cup L^{2}\left(I_{N, N}^{n}\right)\right) .
$$

If we send $N \rightarrow \infty$, then $B_{I}^{(n)}$ must live on the one-dimensional diagonal in $V_{n} \otimes$ $L^{2}\left(\mathbb{R}^{n}\right)$, which is only possible for $n=0$ and $n=1$. Centeredness of our flow excludes $n=0$, and thus we remain only with the possibility that $B_{I} \in L^{2}\left(I, V_{1}\right)$. Additivity of the increments then yields that $B_{I}=\xi \cdot \chi_{I}$ for some locally $L^{2}$-function $\xi$. 
In many interesting cases, the space $V_{1}$ is one-dimensional. In such a situation a corresponding centered flow must be of the form

$$
B_{I}=v \otimes f \cdot \chi_{I}
$$

where $v$ is a fixed unit vector in $V_{1}$ and $f \in L_{\text {loc }}^{2}(I)$. If we now restrict ourselves to selfadjoint stationary normalized flows, then we must have $\varphi\left(B_{[0, t)} B_{[0, t)}\right)=t$ and thus (note that, because of selfadjointness, $f$ is real-valued)

$$
t=\varphi\left(B_{[0, t)} B_{[0, t)}\right)=\left\langle v \otimes f \cdot \chi_{[0, t)}, v \otimes f \cdot \chi_{[0, t)}\right\rangle=\int_{0}^{t}|f(t)|^{2} d t,
$$

i.e. $f$ must be a function with (almost surely) constant modulus 1 .

Theorem 3.3. If the space $V_{1}$ in Theorem 3.2 is one-dimensional, then every selfadjoint stationary normalized flow $\left(B_{I}\right)_{I \in \mathcal{I}}$ has the same distribution for $B_{[0,1)}$. Thus, within the class of generalized Brownian motions with one-dimensional space $V_{1}$, the distribution for $I=[0,1)$ of the generating flow $\left(v \otimes \chi_{I}\right)_{I \in \mathcal{I}}$ is an invariant of the corresponding continuous Bernoulli systems with respect to filtration preserving isomorphisms.

Note that the distribution of $v \otimes \chi_{I}$ for arbitrary $I$ is just a dilation of the distribution for $I=[0,1)$, thus does not contain any additional information.

Proof. A filtration preserving isomorphism between two continuous Bernoulli systems maps a selfadjoint stationary normalized flow to an object of the same kind. For a generalized Brownian motion, the generating flow $\left(v \otimes \chi_{I}\right)_{I \in \mathcal{I}}$ is always selfadjoint, stationary and normalized. On the other side, as we have seen above, every selfadjoint stationary normalized flow must be of the form $v \otimes f \chi_{I}$, where $f$ is a function of constant modulus 1 . However, in the calculation of moments for such operators, only the inner product between the involved functions will play a role, which means that the moments of $\left(v \otimes \chi_{I}\right)_{I \in \mathcal{I}}$ are the same as those of $\left(v \otimes f \chi_{I}\right)_{I \in \mathcal{I}}$. Thus the moments of the generating flows of two generalized Brownian motions must be mapped onto each other by a filtration preserving isomorphism.

Corollary 3.4. 1) The $q$-Brownian motions (with $-1 \leq q \leq 1$ ) of BSp1, BKS] lead for different $q$ to non-isomorphic continuous Bernoulli systems.

2) The generalized Brownian motions with weight function $t_{q}$ of BSp2 lead for different $q$ to non-isomorphic continuous Bernoulli systems.

Proof. Both cases fit into the frame of generalized Brownian motions, and it is easy to see that their space $V_{1}$ is one-dimensional. Thus the distribution of the underlying Brownian motions distinguishes these objects with respect to filtration preserving isomorphisms. It is easy to see that all distributions are different.

\section{Moments of QUantum LÉvy PROCESSES}

Important information about stationary flows $\left(B_{I}\right)_{I \in \mathcal{I}}$ is contained in moments of the corresponding quantum Lévy processes.

Lemma 4.1. Let $B=\left(B_{I}\right)_{I \in \mathcal{I}}$ be a stationary flow and $\left(B_{t}\right)_{t \geq 0}$ the corresponding quantum Lévy process. Then there exist constants $\alpha, \beta$, and $\gamma$ such that we have 
for all $t>0$

$$
\begin{aligned}
\varphi\left(B_{t}\right) & =\alpha t, \\
\varphi\left(B_{t}^{2}\right) & =\alpha^{2} t^{2}+\beta t, \\
\varphi\left(B_{t}^{3}\right) & =\alpha^{3} t^{3}+3 \alpha \beta t^{2}+\gamma t .
\end{aligned}
$$

Proof. For all $s, t \geq 0$, we have

$$
B_{s+t}=B_{[0, s)}+B_{[s, s+t)},
$$

and thus

$$
\varphi\left(B_{s+t}\right)=\varphi\left(B_{s}\right)+\varphi\left(B_{t}\right),
$$

which gives, by continuity, the equation for the first moment, with $\alpha=\varphi\left(B_{1}\right)$.

For the second moment we get

$$
B_{s+t}^{2}=B_{[0, s)}^{2}+B_{[0, s)} \cdot B_{[s, s+t)}+B_{[s, s+t)} \cdot B_{[0, s)}+B_{[s, s+t)}^{2} .
$$

Pyramidal independence gives

$$
\varphi\left(B_{s+t}^{2}\right)=\varphi\left(B_{s}^{2}\right)+\varphi\left(B_{t}^{2}\right)+2 \varphi\left(B_{s}\right) \varphi\left(B_{t}\right),
$$

which implies the equation for the second moment.

Similarly, one shows the result for the third moment.

Note that pyramidal independence does not allow us to calculate all mixed moments of fourth and higher order: e.g., we cannot make a general statement about $\varphi\left(B_{[0, s)} B_{[s, s+t)} B_{[0, s)} B_{[s, s+t)}\right)$. Thus, in this generality, similar statements as in Lemma 4.1 are not accessible for higher moments. Nevertheless, explicit polynomial bounds for the growth of higher moments are established in Kös1, Kös4, Kös2, as an application of Burkholder-Gundy resp. Burkholder/Rosenthal inequalities for non-commutative $L^{p}$-martingales [PX1, JX].

However, if we require some more special structure, then we can say much more about the behaviour of higher moments. In this section we want to consider the case where we have an order invariance of the moments of the increments, in the sense that such moments do not change if we shift the increments against each other, as long as we do not change the relative position of the intervals. Let us first consider a discrete version of this before we treat the continuous case.

4.2. Limit theorem for order invariant distributions. Consider random variables $b_{i}^{(N)}(i, N \in \mathbb{N}, i \leq N)$ living in some non-commutative probability space $(\mathcal{A}, \varphi)$.

For an $n$-tuple

$$
\mathbf{i}:\{1, \ldots, n\} \rightarrow\{1, \ldots, N\}
$$

we put

$$
b_{\mathbf{i}}^{(N)}=b_{i(1)}^{(N)} \cdots b_{i(n)}^{(N)} .
$$

For an $\mathbf{i}$ as above, we denote by $|\mathbf{i}|$ the number of elements in the range of $\mathbf{i}$.

Definition 4.3. 1) Let $\mathbf{i}, \mathbf{j}:\{1, \ldots, n\} \rightarrow \mathbb{N}$ be two $n$-tuples of indices. We say that they are order equivalent, denoted by $\mathbf{i} \sim \mathbf{j}$, if

$$
i(k) \leq i(l) \Longleftrightarrow j(k) \leq j(l) \quad \text { for all } k, l=1, \ldots, n .
$$


We denote by $\mathcal{O}(n)$ the set of equivalence classes for maps $\mathbf{i}:\{1, \ldots, n\} \rightarrow \mathbb{N}$ under this order equivalence. Note that for each $n$ this is a finite set.

2) We say that the distribution of the variables $b_{i}^{(N)}$ is order invariant if we have for all $n, N \in \mathbb{N}$ and all $\mathbf{i}, \mathbf{j}:\{1, \ldots, n\} \rightarrow\{1, \ldots, N\}$ with $\mathbf{i} \sim \mathbf{j}$ that

$$
\varphi\left(b_{\mathbf{i}}^{(N)}\right)=\varphi\left(b_{\mathbf{j}}^{(N)}\right) .
$$

In this case we denote, for $\sigma \in \mathcal{O}(n)$, by $\varphi\left(b_{\sigma}^{(N)}\right)$ the common value of $\varphi\left(b_{\mathbf{i}}^{(N)}\right)$ for $\mathbf{i} \in \sigma$.

Given such order invariant random variables, one can make quite precise statements about the moments of the sums $b_{1}^{(N)}+\cdots+b_{N}^{(N)}$ in the limit $N \rightarrow \infty$. The proof of this limit theorem follows the usual arguments, see, e.g., $\mathrm{SpW}$, and we will omit the proof.

Theorem 4.4. Consider random variables $b_{i}^{(N)} \in(\mathcal{A}, \varphi)(i, N \in \mathbb{N}, i \leq N)$, whose distribution is order invariant. Assume that for all $n \in \mathbb{N}$ and all $\sigma \in \mathcal{O}(n)$ the following limit exists:

$$
c(\sigma):=\lim _{N \rightarrow \infty} N^{|\sigma|} \varphi\left(b_{\sigma}^{(N)}\right) .
$$

Define

$$
S_{N}:=b_{1}^{(N)}+\cdots+b_{N}^{(N)} .
$$

Then we have for all $n \in \mathbb{N}$

$$
\lim _{N \rightarrow \infty} \varphi\left(S_{N}^{n}\right)=\sum_{\sigma \in \mathcal{O}(n)} \alpha_{\sigma} c(\sigma),
$$

where the $\alpha_{\sigma}$ are the constants,

$$
\alpha_{\sigma}=\lim _{N \rightarrow \infty} \frac{\#\{\mathbf{i}:\{1, \ldots, n\} \rightarrow\{1, \ldots, N\} \mid \mathbf{i} \in \sigma\}}{N^{|\sigma|}}=\frac{1}{|\sigma| !} .
$$

4.5. Moments of order invariant quantum Lévy processes. In the following, we will use, for two intervals $I, J \in \mathcal{I}$, the notation $I<J$ to indicate that we have $s<t$ for all $s \in I$ and $t \in J$.

Definition 4.6. Let $\left(B_{I}\right)_{I \in \mathcal{I}}$ be a flow. We say that the flow (or its corresponding quantum Lévy process) is order invariant if we have for all $I_{1}, \ldots, I_{n} \in \mathcal{I}$ with $I_{k} \cap I_{l}=\emptyset(k, l=1, \ldots, n)$ that

$$
\varphi\left(B_{I_{1}} \cdots B_{I_{n}}\right)=\varphi\left(B_{I_{1}+t_{1}} \cdots B_{I_{n}+t_{n}}\right)
$$

for all $t_{1}, \ldots, t_{n}$ with the property that, for all $k, l=1, \ldots, n, I_{k}<I_{l}$ implies $I_{k}+t_{k}<I_{l}+t_{l}$.

Remark 4.7. Note that an order invariant flow is in particular stationary.

Now consider such an order invariant flow $\left(B_{I}\right)_{I \in \mathcal{I}}$. Put

$$
b_{i}^{(N)}:=B_{\left[\frac{i-1}{N}, \frac{i}{N}\right)} .
$$

Then we have

$$
S_{N}=b_{1}^{(N)}+\cdots+b_{N}^{(N)}=B_{1}
$$


for all $N \in \mathbb{N}$ and, since the distribution of the $b_{i}^{(N)}$ is order invariant, our limit theorem, Theorem 4.4 yields that

$$
\varphi\left(B_{1}^{n}\right)=\sum_{\sigma \in \mathcal{O}(n)} \alpha_{\sigma} c(\sigma)
$$

if all

$$
c(\sigma):=\lim _{N \rightarrow \infty} N^{|\sigma|} \varphi\left(b_{\sigma}^{(N)}\right)
$$

exist.

Proposition 4.8. Let $\left(B_{I}\right)_{I \in \mathcal{I}}$ be an order invariant flow. Then, for all $n \in \mathbb{N}$ and $\sigma \in \mathcal{O}(n)$, the limit

$$
c(\sigma):=\lim _{N \rightarrow \infty} N^{|\sigma|} \varphi\left(b_{\sigma}^{(N)}\right)
$$

exists.

Proof. We will prove this, for fixed $n$, by induction over the length of $\sigma$, starting with maximal length of $\sigma$.

Namely, fix $n$ and consider first a $\sigma$ with $|\sigma|=n$. This means that $\mathbf{i}=$ $(i(1), \ldots, i(n)) \in \sigma$ is a tuple of $n$ different numbers. By using the stochastic independence we get

$$
\begin{aligned}
N^{|\sigma|} \varphi\left(b_{\sigma}^{(N)}\right) & =N^{n} \varphi\left(b_{i(1)}^{(N)} \cdots b_{i(n)}^{(N)}\right) \\
& =N^{n} \varphi\left(b_{i(1)}^{(N)}\right) \cdots \varphi\left(b_{i(n)}^{(N)}\right) \\
& =N^{n} \varphi\left(b_{1}^{(N)}\right)^{n} \\
& =\left(N \varphi\left(B_{\left[0, \frac{1}{N}\right)}\right)\right)^{n} \\
& =\varphi\left(B_{1}\right)^{n},
\end{aligned}
$$

and hence the limit

$$
c(\sigma):=\lim _{N \rightarrow \infty} N^{|\sigma|} \varphi\left(b_{\sigma}^{(N)}\right)=\varphi\left(B_{1}\right)^{n}
$$

exists.

Now consider an arbitrary $\sigma \in \mathcal{O}(n)$ and assume that we have proved the existence of the limits $c\left(\sigma^{\prime}\right)$ for all $\sigma^{\prime} \in \mathcal{O}(n)$ with $\left|\sigma^{\prime}\right|>|\sigma|$. Choose an $n$-tuple $\mathbf{i}=(i(1), \ldots, i(n)) \in \sigma$ and consider

$$
\varphi\left(B_{[i(1), i(1)+1)} \cdots B_{[i(n), i(n)+1)}\right) .
$$

Again, we decompose the intervals of length 1 into $N$ subintervals of length $1 / N$, so that we can write this also as

$$
\varphi\left(\left(\sum_{k(1)=1}^{N} B_{\left[i(1)+\frac{k(1)-1}{N}, i(1)+\frac{k(1)}{N}\right)}\right) \cdots\left(\sum_{k(n)=1}^{N} B_{\left[i(n)+\frac{k(n)-1}{N}, i(n)+\frac{k(n)}{N}\right)}\right)\right) .
$$

If we multiply this out and collect terms together with the same relative position of the subintervals, then we get a sum of terms, one of which is exactly $N^{|\sigma|} \varphi\left(b_{\sigma}^{(N)}\right)$, and the others are of the form $\gamma_{\sigma^{\prime}} \varphi\left(b_{\sigma^{\prime}}^{(N)}\right)$, for $\sigma^{\prime}$ with $\left|\sigma^{\prime}\right|>|\sigma|$. Since also $\gamma_{\sigma^{\prime}} \sim$ $N^{\left|\sigma^{\prime}\right|}$ for $N \rightarrow \infty$, we know by our induction hypothesis that all these other terms have a finite limit for $N \rightarrow \infty$. Since the left hand side of our equation does not depend on $N$, the term $N^{|\sigma|} \varphi\left(b_{\sigma}^{(N)}\right)$ must also have a finite limit for $N \rightarrow \infty$. 
Of course, the same argument works if we replace the time 1 by an arbitrary time $t$. In this case, we get the existence of the limits

$$
c_{t}(\sigma):=\lim _{N \rightarrow \infty} N^{|\sigma|} \varphi\left(B_{[i(1), i(1)+t / N)} \cdots B_{[i(n), i(n)+t / N)}\right),
$$

for $(i(1), \ldots, i(n)) \in \sigma$. The remaining question is how these $c_{t}(\sigma)$ depend on the time $t$.

Lemma 4.9. We have that

$$
c_{s}(\sigma)=c(\sigma) \cdot s^{|\sigma|} \quad \text { for all } s \in \mathbb{Q} .
$$

Proof. For $(i(1), \ldots, i(n)) \in \sigma$ and $t \in \mathbb{R}$, we have

$$
\begin{aligned}
c_{2 t}(\sigma)= & \lim _{N \rightarrow \infty} N^{|\sigma|} \varphi\left(B_{[i(1), i(1)+2 t / N)} \cdots B_{[i(n), i(n)+2 t / N)}\right) \\
= & \lim _{N \rightarrow \infty} N^{|\sigma|} \varphi\left(\left(B_{[i(1), i(1)+t / N)}+B_{[i(1)+t / N, i(1)+2 t / N)}\right)\right. \\
& \left.\quad \cdots\left(B_{[i(n), i(n)+t / N)}+B_{[i(n)+t / N, i(n)+2 t / N)}\right)\right) \\
= & 2^{|\sigma|} c_{t}(\sigma) .
\end{aligned}
$$

Note that for each block of $\sigma$ we can choose either the increments from $i$ to $i+t / N$ or the increments from $i+t / n$ to $i+2 t / N$ to match up, i.e. each block of $\sigma$ contributes a factor 2 . On the other hand, terms which match for some block an increment from $i$ to $i+t / N$ with an increment from $i+t / N$ to $i+2 t / N$ vanish in the limit, because they correspond to a $\sigma^{\prime}$ with $\left|\sigma^{\prime}\right|>|\sigma|$, and so they have to be multiplied with a higher power of $N$ to give a non-trivial limit.

In the same way as above one can also see that for any $k \in \mathbb{N}$ and any $t \in \mathbb{R}$ we have

$$
c_{k t}(\sigma)=k^{|\sigma|} c_{t}(\sigma)
$$

This finally yields the assertion.

By invoking different $t$ for each block of $\sigma$ one could also derive functional equations for these quantities which, together with the fact that they are measurable, would extend the statement of the above lemma to all $t \in \mathbb{R}$. However, we do not need this because the continuity of the moments $\varphi\left(B_{t}^{n}\right)$ allows us to extend the statement in the next theorem directly from rational to all real times $t$.

Let us summarize in the following theorem our results.

Theorem 4.10. Let $\left(B_{t}\right)_{t \geq 0}$ be an order invariant quantum Lévy process. Then there exist constants $c(\sigma)$ for all $\sigma \in \mathcal{O}$ such that we have for all $n \in \mathbb{N}$ and all $t \geq 0$

$$
\varphi\left(B_{t}^{n}\right)=\sum_{\sigma \in \mathcal{O}(n)} \frac{1}{|\sigma| !} c(\sigma) t^{|\sigma|} .
$$

In the next section we will see that quantum Lévy processes which are not order invariant do not necessarily have such a polynomial behaviour of their moments.

4.11. An example of a non-order invariant generalized Brownian motion. Finally, we want to present an example of a quantum Lévy process which is not order invariant. This example is a generalization of the $q_{i j}$-relations

$$
a_{i} a_{j}^{*}-q_{i j} a_{j}^{*} a_{i}=\delta_{i j} \cdot 1
$$


to the continuous case, and it is formally given by

$$
a_{t} a_{s}^{*}-q(s-t) a_{s}^{*} a_{t}=\delta(s-t) \cdot 1 .
$$

This situation can be realized rigorously as follows: Put $\mathcal{H}:=L^{2}(\mathbb{R})$, and consider on $\mathcal{H} \otimes \mathcal{H}=L^{2}\left(\mathbb{R}^{2}\right)$ the operator $T$, given by

$$
(T f)(s, t)=q(s, t) \cdot f(t, s),
$$

where $q=q(\cdot, \cdot)$ is a fixed function of two variables. This $T$ fulfills the braid relations. If we assume in addition that $q$ has the properties $\bar{q}(s, t)=q(t, s)$ and $|q(s, t)| \leq 1$ for all $s, t$, then $T$ is also selfadjoint and contractive. Thus the assumptions of $[\mathrm{BSp} 3$ are fulfilled and the corresponding Fock space construction yields a positive inner product and, for each $f \in \mathcal{H}$, creation and annihilation operators $d^{*}(f)$ and $d(f)$, respectively. Put now, for $I \in \mathcal{I}$,

$$
B_{I}:=d\left(\chi_{I}\right)+d^{*}\left(\chi_{I}\right)
$$

and define

$$
\begin{array}{rlrl}
\mathcal{A} & :=\mathrm{vN}\left(B_{I} \mid I \in \mathcal{I}\right), & \\
\mathcal{A}_{I} & :=\operatorname{vN}\left(B_{J} \mid J \in \mathcal{I}, J \subset I\right) & & (I \in \mathcal{I}), \\
\varphi(a) & :=\langle a \Omega, \Omega\rangle & (a \in \mathcal{A}) .
\end{array}
$$

If $q$ is real (and thus symmetric, i.e. $q(s, t)=q(t, s)$ ), then $\varphi$ is a faithful trace on $\mathcal{A}$. Furthermore, if $q$ is stationary, i.e. $q(s, t)=q(s-t)$, then one has a well-defined second quantization $\Gamma\left(S_{t}\right)$ (see [Kro] $)$ of the usual shift $(u \in \mathbb{R})$

$$
S_{u}: L^{2}(\mathbb{R}) \rightarrow L^{2}(\mathbb{R}), \quad\left(S_{u} f\right)(t)=f(t-u),
$$

which is compatible with the filtration of the von Neumann algebras. Let us summarize this in the following proposition.

Proposition 4.12. Let $q: \mathbb{R} \rightarrow \mathbb{R}$ be a measurable function with the property

$$
-1 \leq q(t)=q(-t) \leq 1 \quad \text { for all } t \in \mathbb{R} .
$$

Then $\left(\mathcal{A}, \varphi,\left(\mathcal{A}_{I}\right)_{I \in \mathcal{I}}\right)$ corresponding to the operator $T$ on $L^{2}\left(\mathbb{R}^{2}\right)$ given by

$$
(T f)(s, t)=q(s-t) \cdot f(t, s)
$$

forms a continuous Bernoulli system with corresponding stationary flow

$$
B_{I}:=d\left(\chi_{I}\right)+d^{*}\left(\chi_{I}\right) \quad(I \in \mathcal{I}) .
$$

If $q$ is constant, then one recovers the example of the $q$-Brownian motion BSp1, BKS, which is of course order invariant. If, however, $q$ is not constant, then this flow is not order invariant. For example, by using the definition of the operators $d(f)$ and $d^{*}(f)$, one readily finds for $I, J \in \mathcal{I}$ with $I \cap J=\emptyset$ that

$$
\varphi\left(B_{I} B_{J} B_{I} B_{J}\right)=\int_{I} \int_{J} q(s-t) d s d t,
$$

which gives for the fourth moment of our quantum Lévy process

$$
\varphi\left(B_{t}^{4}\right)=t^{2}+\int_{0}^{t} q\left(t_{1}-t_{2}\right) d t_{1} d t_{2} .
$$


(Note that formally these results can be obtained by using the Ito formula

$$
\left.d B_{s} d B_{t} d B_{s} d B_{t}=q(s-t) d s d t .\right)
$$

By making different choices of the function $q$, this shows that there is quite a variation of the behaviour of the fourth (and higher) moments for non-order invariant quantum Lévy processes.

\section{REFERENCES}

[Arv] W. Arveson, Non-commutative Dynamics and E-semigroups. Springer Monographs in Mathematics, Springer-Verlag, 2003. MR1978577 (2004g:46082)

[BKS] M. Bożejko, B. Kümmerer, and R. Speicher, q-Gaussian processes: non-commutative and classical aspects. Commun. Math. Phys. 185 (1997), 129-154. MR1463036 (98h:81053)

[BSp1] M. Bożejko, and R. Speicher, An example of a generalized Brownian motion. Commun. Math. Phys. 137 (1991), 519-531. MR.1105428 (92m:46096)

[BSp2] M. Bożejko, and R. Speicher, Interpolations between bosonic and fermionic relations given by generalized Brownian motions. Math. Z. 222 (1996), 135-160. MR1388006 (97g:81031)

[BSp3] M. Bożejko, and R. Speicher, Completely positive maps on Coxeter groups, deformed commutation relations, and operator spaces. Math. Ann. 300 (1994), 97-120. MR1289833 (95g:46105)

[GM1] M. Guta, and H. Maassen, Symmetric Hilbert spaces arising from species of structures. Math. Z. 239 (2002), 477-513. MR.1893849 (2003g:46085)

[GM2] M. Guta, and H. Maassen, Generalised Brownian motion and second quantisation. J. Funct. Anal. 191 (2002), 241-275. MR1911186 (2003d:46084)

[HKK] J. Hellmich, C. Köstler, and B. Kümmerer: Non-commutative continuous Bernoulli shifts. arXiv:math.OA/0411565 v1, 2004.

[JX] M. Junge, and Q. Xu: Non-commutative Burkholder/Rosenthal inequalities. Ann. Probab. 31 (2003), no.2., 948-995. MR1964955 (2004f:46078)

[Kös1] C. Köstler, Quanten-Markoff-Prozesse und Quanten-Brownsche Bewegungen. Ein operatoralgebraischer Zugang. Ph.D. Thesis, Stuttgart (2000).

[Kös2] C. Köstler, A quantum stochastic extension of Stone's Theorem. In Advances in Quantum Dynamics (South Hadley, MA, 2002), 209-222, Contemp. Math. 335, Amer. Math. Soc., Providence, RI, 2003. MR2029625 (2004k:46109)

[Kös3] C. Köstler, On the relationship of continuous Bernoulli systems, Tsirelson's continuous products of probability spaces and Arveson's product systems. In preparation.

[Kös4] C. Köstler, An operator algebraic approach to quantum Lévy processes and quantum Brownian motions. Preprint.

[Kro] I. Krölak, Contractivity properties of Ornstein-Uhlenbeck semigroup for general commutation relations. Math. Z. 250 (2005), 915-937. MR2180382(2006i:81105)

[Küm1] B. Kümmerer, Markov dilations on $W^{*}$-algebras. J. Funct. Anal. 63 (1985), 139-177. MR0803091 (87b:46070)

[Küm2] B. Kümmerer, Quantum white noise. In Infinite Dimensional Harmonic Analysis (Tübingen, 1995), 156-168. Graebner, Tübingen, 1996. MR1406579 (97f:46101)

[Pop] S. Popa, Orthogonal pairs of *-subalgebras in finite von Neumann algebras. J. Operator Theory 9 (1983), 253-268. MR0703810 (84h:46077)

[PX1] G. Pisier, and Q. Xu, Non-commutative martingale inequalities. Commun. Math. Phys. 189 (1997), 667-698. MR1482934 (98m:46079)

[PX2] G. Pisier, and Q. Xu, Non-commutative $L^{p}$-spaces. In Handbook of the geometry of Banach spaces, Vol. 2, 1459-1517, North Holland, Amsterdam, 2003. MR.1999201 (2004i:46095)

[SpW] R. Speicher, and W. von Waldenfels, A general central limit theorem and invariance principle. In Quantum Probability and Related Topics IX (1994), 371-387.

[Tsi] B. Tsirelson, Nonclassical stochastic flows and continuous products. Prob. Surv. 1 (2004), 173-298 (electronic). MR2068474 (2005m:60080) 
[Voi] D. Voiculescu, Free entropy. Bull. London Math. Soc. 34 (2002), 257-278. MR1887698 (2003c:46077)

[VDN] D. Voiculescu, K. Dykema, and A. Nica, Free Random Variables. AMS, Providence, Rhode Island, 1992. MR1217253 (94c:46133)

School of Mathematics and Statistics, Herzberg Bullding, Carleton University, OttaWA, Ontario, K1S 5B6 Canada

E-mail address: koestler@math.carleton.ca

Department of Mathematics and Statistics, Jeffery Hall, Queen's University, Kingston, Ontario, K7L 3N6 Canada

E-mail address: speicher@mast.queensu.ca 